\title{
ON INVERSION OF GRADIENT MAGNETIC DATA FOR DETECTION OF MULTIPLE BURIED METALLIC OBJECTIVES
}

\author{
Dr. Ruizhong Jia, PetRos EiKon, Concord, Ontario, Canada \\ Dr. R. W. Groom, PetRos EiKon, Concord, Ontario, Canada
}

\begin{abstract}
In our previous work (R.W. Groom, R. Jia And C. Alvarez 2003), we have developed algorithms to implement an Euler depth estimator as well as an inversion algorithm for detecting a simple dipole, which is often a suitable model for UXO applications. These algorithms worked independently with a single anomaly. We have combined these algorithms to determine the locations as well as the internal magnetization vectors of buried objects in a practical field survey. We start with the Euler deconvolution depth estimator that gives the locations of buried objects using the measured total field and its measured/calculated gradients. Based on these initial results, a subset of measured data is selected and a local search grid is set for each individual body. Then the magnetization inversion algorithm is utilized to find the locations and internal magnetization vectors of the buried bodies. The inversion process involves performing an automatic iterative grid volume modification according to a prescribed volume range of the buried objectives. Consequently we can determine the location and internal magnetization vector of each individual body by applying the inversion algorithm in an automatic way. In general, the locations identified in this way are more accurate due to the fact that only the measured total field data is used in the later stage. It is shown that Magnetization Vector Inversion is relatively insensitive to data density and thus works more stably. Furthermore, starting with good Euler solutions is essential to guarantee an appropriate selection of dataset that incorporates substantial variation of the field and field gradients of each object.
\end{abstract}

\section{Introduction}

To investigate imaging, inversion and discrimination of metallic objects within the ground with the use of magnetic data collected on the surface; we decided to proceed first by applying the standard Euler deconvolution depth estimator. Euler deconvolution depth estimator is a powerful method to gain preliminary information on position, shape, and depth of causative bodies from gravity and magnetic fields (Reid et al., 1990, Changyou Zhang et al, 2000). The total field and its gradients have to be provided for this technique. In a real survey in which the high density total field dataset is collected, we may grid the data to generate the derivatives of the total field by FFT.

By setting appropriate size of a moving window that is appropriate to incorporate substantial variation of the field and field gradients of causative bodies individually, we may find all the possible locations of buried objectives by Euler deconvolution depth estimator. Geometrically, Euler solutions form broad clouds rather than dense clusters, making it difficult to outline causative sources. We utilize a post processing technique, the Rodin algorithm (V. Mikehailov et al, 2003), to eliminate some unrealistic solutions. Based on the spatial separation of the buried objectives, we then apply a technique to split the solutions thus obtained into groups, each of which forms a dense cluster which clearly outline possible causative sources. Finally, the location of each individual body is determined by means of statistics. Our imaging techniques enable us to visualize the Euler solutions in a three dimensional coordinate system. 
Once the Euler solutions of the buried objectives are provided, they serve as the initial solutions for performing the magnetization inversion algorithm.. The inversion process proceeds in an automatic way. A local search grid is set for each individual body and a subset of measured total data that incorporates substantial variation of the field and field gradients of the body is selected. By performing an automatic iterative grid volume modification according to a prescribed volume range of the buried objectives, optimum solutions giving the locations as well as the internal magnetization vectors of buried objects are produced in terms of minimizing the $\chi^{2}$ error functional.

\section{Euler Deconvolution Depth Estimator}

\section{Calculating Derivatives of total field by FFT}

In order to utilize Euler deconvolution depth estimator, horizontal and vertical derivatives have to be either measured or calculated. In the case that only total field is measured, horizontal and vertical derivatives must be computed. The inline derivative or transverse derivative can be computed by either simple difference or FFT. To use FFT, a gridded magnetic dataset has to be prepared from which the vertical derivative can be obtained via the formula

$$
F\left[\left.\frac{\partial T}{\partial z}\right|_{\left(x, y, z_{0}\right)}\right]=\left(\sqrt{k_{x}^{2}+k_{y}^{2}}\right) F\left[T\left(x, y, z_{0}\right)\right],
$$

where $F[]$ denote the 2 dimensional Fourier transform, $k_{x}$ and $k_{y}$ are the wave numbers along $\mathrm{X}$-axis and $\mathrm{Y}$-axis respectively, $z_{0}$ is the altitude at which the survey is carried out. FFT is a useful technique to compute gradients, especially the vertical gradients

\section{Test Results for First Survey}

We illustrate the techniques with two synthetic surveys. In the first survey, we utilize 60 profiles of length $30 \mathrm{~m}$ along the EW direction separated by $0.5 \mathrm{~m}$ with a data sampling every $0.5 \mathrm{~m}$. In the second survey, we utilize 30 profiles of length $30 \mathrm{~m}$ along the EW direction separated by $1 \mathrm{~m}$ with a data sampling every $1 \mathrm{~m}$.

Three small objects, each having dimension $20 \mathrm{~cm}$ x $20 \mathrm{~cm}$ x $20 \mathrm{~cm}$ and constant magnetization, were inserted in the survey region. The locations and the orientation of the internal magnetization vectors of these objects are given in Table 1. The first row indicates the object number, center, the magnetization vector and volume. The strength is with respect to the earth's field. In both surveys, the background earth field is set as:

$$
\begin{gathered}
\text { Inclination }=10 \text { degree, } \\
\text { Declination }=75 \text { degree, } \\
\text { Intensity }=52500 \mathrm{nT} .
\end{gathered}
$$

\begin{tabular}{|c|c|c|c|c|c|c|c|}
\hline Body & $\mathrm{X}$ center & Y center & $\mathbf{Z}$ center & dip & decl & strength & Cell Size \\
\hline 1 & 0 & 8 & -3 & 45 & 45 & 6 & 0.008 \\
\hline 2 & -8 & --8 & -2.5 & 80 & 120 & 7 & 0.008 \\
\hline 3 & 8 & -8 & -2 & 35 & 70 & 7 & 0.008 \\
\hline
\end{tabular}

Table 1: Objectives in survey 
We first generated Euler solutions for Survey 1. To utilize Euler deconvolution depth estimator, the moving window size is set $5.5 \mathrm{~m}$ by $5.5 \mathrm{~m}$. We produced solutions with true total field data and gradients. The filtered Euler solutions by Rodin algorithm was displayed in Figure 1. The filtered solutions form dense clusters outlining the objects. In order to make the data more realistic, we added $0.5 \mathrm{nT}$ random noise to the true total field and then interpolated the data on a 64 by 64 grid (grid cell size $0.45 \mathrm{~m}$ by $0.45 \mathrm{~m}$ ). With this gridded dataset the gradients of total field was computed by FFT. Figure 2 shows the true vertical derivative and the estimated vertical derivative of total field by FFT along a particular grid line. It is seen that FFT gradients agrees well with the true value. Then solutions generated with this data set were produced. Finally, we also experimented with the noisy total field and its FFT vertical gradient and simple difference horizontal derivatives. All these results are presented in Table 2.

We see from table 2 that if the total field and its derivatives are known accurately, Euler deconvolution depth estimator is a rapid and accurate technique for object location. In this particular example, reasonably good results were produced even with noisy total field data and estimated FFT or simple gradients. For FFT gradients, both the horizontal positions and depth estimates are made to be within $0.4 \mathrm{~m}$ of the known values. With simple difference horizontal derivatives, the horizontal positions are made to be within $0.2 \mathrm{~m}$ of the true value and depth estimates are within $0.5 \mathrm{~m}$ of the known depths of burial. For both cases, the depth estimates are within approximately $15 \%$ of the sensor to source distance.

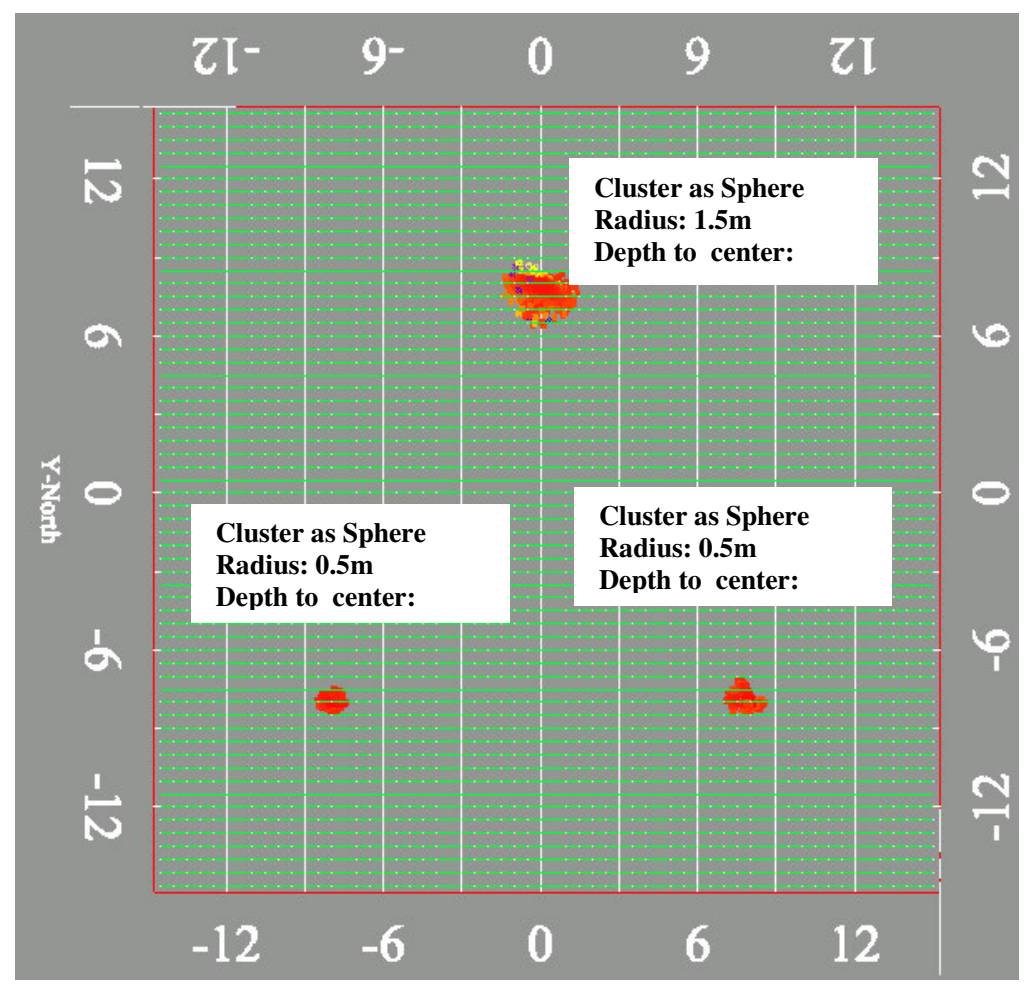

\section{Figure 1: Filtered Euler Solutions}

Filtered Euler solutions with Rodin algorithm, where Euler solutions were produced with true total field data and true gradients 


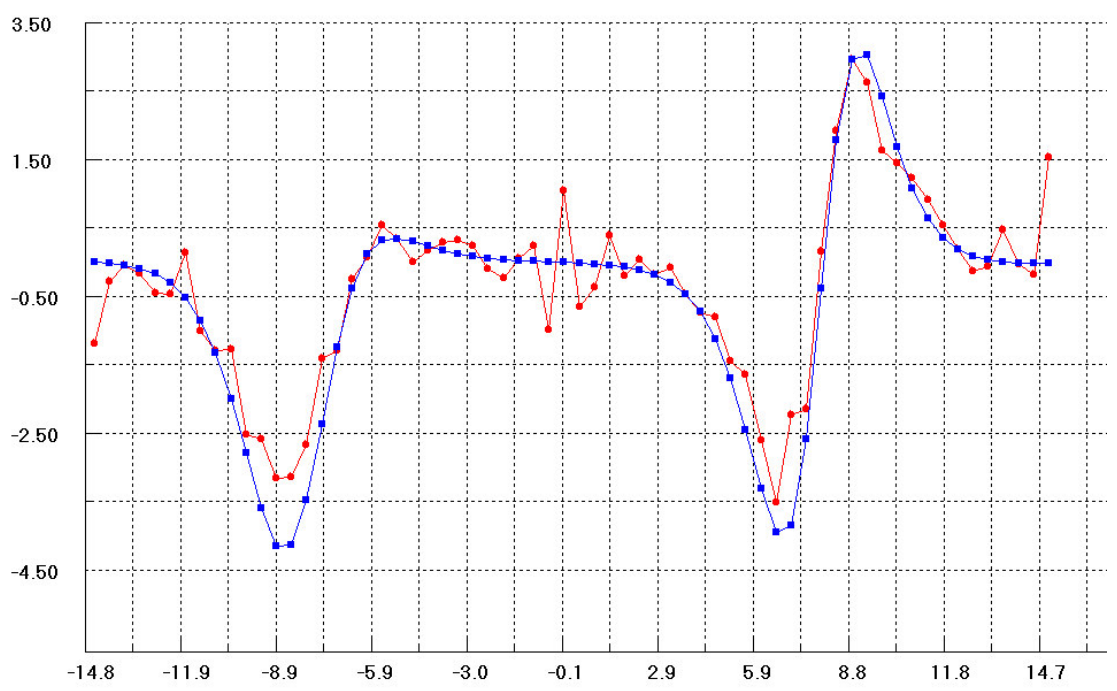

Figure 2 FFT vertical derivative VS. true vertical derivative Blue curve: true vertical derivative of total field

Red curve: estimated FFT vertical derivative on a 64 x 64 grid

Table 2 Euler Solutions for Survey 1

\begin{tabular}{|c|c|c|c|c|}
\hline & Body & $\mathrm{X}$ center & Y center & $\mathbf{Z}$ center \\
\hline \multirow[t]{3}{*}{ Actual locations } & 1 & 0 & 8 & -3 \\
\hline & 2 & -8 & -8 & -2.5 \\
\hline & 3 & 8 & -8 & -2 \\
\hline \multirow[t]{3}{*}{ true Btotal and true gradients } & 1 & 0.01 & 7.87 & -3.10 \\
\hline & 2 & -7.97 & -7.97 & -2.48 \\
\hline & 3 & 7.98 & -7.99 & -1.97 \\
\hline \multirow[t]{3}{*}{ noisy Btotal and FFT gradients } & 1 & -0.01 & 7.59 & -2.64 \\
\hline & 2 & -7.82 & -7.97 & -2.58 \\
\hline & 3 & 7.86 & -7.97 & -2.18 \\
\hline \multirow{3}{*}{$\begin{array}{l}\text { noisy Btotal and FFT vertical } \\
\text { gradients and simple difference } \\
\text { horizontal derivatives }\end{array}$} & 1 & -0.13 & 7.87 & -2.51 \\
\hline & 2 & -7.97 & -7.95 & -2.52 \\
\hline & 3 & 7.92 & -7.80 & -2.15 \\
\hline
\end{tabular}

\section{Test Results for Second Survey}

Likewise, Euler solutions were also produced for Survey 2. Recall that Survey 2 has less data density, that is, the line separation is $1 \mathrm{~m}$ and the data is sampled every $1 \mathrm{~m}$. We still added $0.5 \mathrm{nT}$ random noise to the true total field and then interpolated the data on a 64 by 64 grid (grid cell size $0.45 \mathrm{~m}$ by $0.45 \mathrm{~m}$ ).

It is shown that if total field and its gradients are known accurately, the locations of buried objects can be well determined, regardless of data density. However, with FFT or simple difference derivatives, the results generated with this survey are not as good as Survey 1 simply because of its coarser data sampling rate. 
Table 3: Euler Solutions for Survey 2

\begin{tabular}{|c|c|c|c|c|}
\hline \multirow{2}{*}{ Actual locations } & \multicolumn{1}{c}{ Body } & X center & Y center & Z center \\
& 1 & 0 & 8 & -3 \\
\cline { 2 - 5 } & 2 & -8 & --8 & -2.5 \\
\cline { 2 - 5 } & 3 & 8 & -8 & -2 \\
\hline \multirow{2}{*}{ true Btotal and true gradients } & 1 & 0.01 & 7.91 & -3.0 \\
\cline { 2 - 5 } & 2 & -7.99 & -7.98 & -2.51 \\
\cline { 2 - 5 } & 3 & 7.99 & -8.0 & -1.96 \\
\hline noisy Btotal and FFT gradients & 1 & -0.75 & 7.17 & -2.34 \\
\cline { 2 - 5 } & 2 & -8.01 & -7.59 & -2.78 \\
\cline { 2 - 5 } & 3 & 7.8 & -7.59 & -2.45 \\
\hline \multirow{2}{*}{$\begin{array}{c}\text { noisy Btotal and FFT vertical } \\
\text { gradients and simple difference } \\
\text { horizontal derivatives }\end{array}$} & 1 & -1.0 & 7.28 & -2.12 \\
\cline { 2 - 5 } & 2 & -8.01 & -7.64 & -2.62 \\
\cline { 2 - 5 } & 3 & 8.13 & -7.80 & -2.35 \\
\hline
\end{tabular}

\section{Magnetization Vector Inversion}

\section{Ridge Regression Analysis for Magnetization Vector Inversion}

As stated in (R.W. Groom, R. Jia And C. Alvarez 2003), the magnetization vector inversion problem can be formulated as

$$
\left|\vec{B}\left(\vec{r}_{i}\right)\right|=\left|\vec{B}_{e}+G\left(\vec{r}_{i}, \vec{r}_{j}\right) \vec{M}_{j} \Delta V_{j}\right|, i=1,2, \ldots, N,
$$

where $G\left(\vec{r}, \vec{r}^{\prime}\right)$ is Green's tensor, $\vec{M}_{j} \Delta V_{j}$ stands for the volume magnetization of cell $j$, and $\vec{r}_{j}$ specifies the location of the center of cell $j, . \vec{B}\left(\vec{r}_{i}\right)$ is the total field at the ith station, $\vec{B}_{e}$ is the earth field. By introducing the notation $\vec{B}\left(\vec{r}_{i}\right)=\left(\begin{array}{c}b_{1}^{i} \\ b_{2}^{i} \\ b_{3}^{i}\end{array}\right), \vec{B}_{e}=\left(\begin{array}{c}b_{1}^{e} \\ b_{2}^{e} \\ b_{3}^{e}\end{array}\right), \vec{M}_{j}=\left(\begin{array}{c}m_{1} \\ m_{2} \\ m_{3}\end{array}\right), G\left(\vec{r}_{i}, \vec{r}_{j}\right) \Delta V_{j}=\left(a_{k l}\right)_{3 \times 3}$, then we have

$$
\begin{aligned}
& m_{1}^{2} \sum_{i=1}^{3} a_{i 1}^{2}+m_{2}^{2} \sum_{i=1}^{3} a_{i 2}^{2}+m_{3}^{2} \sum_{i=1}^{3} a_{i 3}^{2}+2 m_{1} m_{2} \sum_{i=1}^{3} a_{i 1} a_{i 2}+2 m_{1} m_{3} \sum_{i=1}^{3} a_{i 1} a_{i 3}+2 m_{2} m_{3} \sum_{i=1}^{3} a_{i 2} a_{i 3} \\
& +2 m_{1} \sum_{i=1}^{3} a_{i 1} b_{i}^{e}+2 m_{2} \sum_{i=1}^{3} a_{i 2} b_{i}^{e}+2 m_{3} \sum_{i=1}^{3} a_{i 3} b_{i}^{e}+\sum_{i=1}^{3}\left(b_{i}^{e}\right)^{2}=\sum_{i=1}^{3}\left(b_{i}^{k}\right)^{2}, k=1,2, \ldots, N
\end{aligned}
$$

Note that our aim is to determine the vector $\vec{M}_{j}=\left(\begin{array}{l}m_{1} \\ m_{2} \\ m_{3}\end{array}\right)$. 
Obviously, there exist multicolinearities among the unknowns in the linear equation system (1). Therefore, in order to determine $m_{1}, m_{2}, m_{3}$, ridge regression technique has to be utilized, particularly in the case where the field survey is done at low magnetic latitudes.

\section{Test Results with Magnetization Vector Inversion}

We first apply the inversion process to Survey 1. For each Euler solution with noisy total field and FFT gradients in Table 2, we utilized $1 \times 1 \times 1 \mathrm{~m}$ cube centered at the location as a search grid. For each individual object, the total field data is taken from $6 \times 6 \mathrm{~m}$ square centered on xy location of the Euler solution, and the volume range is set between 0.0005 and 0.01 . The inversion process searches for the optimum solution in terms of minimizing the $\chi^{2}$ error functional. The first set of results was generated with true total field data. The second set of results was produced with noisy total field to which $0.5 \mathrm{nT}$ random noise was added. Magnetization Vector Inversion results are illustrated in Table 4. It is shown that with reasonably good measurement of total field, we can well determine the locations and the magnetization vectors, the dip and declination angles in particular.

Table 4: Magnetization Vector Inversion Results for Survey 1

\begin{tabular}{|c|c|c|c|c|c|c|c|c|}
\hline & Body & $X$ center & Y center & $\mathbf{Z}$ center & $\operatorname{dip}$ & decl & strength & Cell Size \\
\hline \multirow[t]{3}{*}{ Actual } & 1 & 0 & 8 & -3 & 45 & 45 & 6 & 0.008 \\
\hline & 2 & -8 & --8 & -2.5 & 80 & 120 & 7 & 0.008 \\
\hline & 3 & 8 & -8 & -2 & 35 & 70 & 7 & 0.008 \\
\hline \multirow{3}{*}{$\begin{array}{l}\text { True total } \\
\text { field }\end{array}$} & 1 & 0.09 & 7.97 & -2.84 & 42.7 & 47.5 & 5.9 & 0.006 \\
\hline & 2 & -8.04 & -8.05 & -2.51 & 79.5 & 144.8 & 17.6 & 0.003 \\
\hline & 3 & 8.01 & -8.0 & -2.03 & 34.8 & 70.6 & 9.7 & 0.007 \\
\hline \multirow{3}{*}{$\begin{array}{l}\text { Noisy total } \\
\text { field }\end{array}$} & 1 & 0.12 & 7.95 & -2.86 & 40.5 & 49.7 & 14.1 & 0.003 \\
\hline & 2 & -8.06 & -8.09 & -2.57 & 79.1 & 150.7 & 31.6 & 0.002 \\
\hline & 3 & 8.01 & -8.0 & -2.03 & 34.2 & 70.9 & 9.8 & 0.006 \\
\hline
\end{tabular}

With Survey 2, we use the Euler solutions with noisy total field and FFT gradients in Table 3. For each individual object, we utilized $1.6 \times 1.6 \times 1.6 \mathrm{~m}$ cube centered at its location as a search grid. Again, the total field data is taken from $6 \times 6 \mathrm{~m}$ square centered on $x y$ location of the Euler solution, and the volume range is set between 0.0005 and 0.01 . The first set of results was generated with true total field data. The second set of results was produced with noisy total field to which $0.5 \mathrm{nT}$ random noise was added. The results are presented in Table 5.

Note that Magnetization Vector Inversion is relatively insensitive to data density, as opposed to Euler Deconvolution Depth estimator. Even with noisy total field data, the location and internal magnetization vector of each object can be well determined. 
Table 5: Magnetization Vector Inversion Results for Survey 2

\begin{tabular}{|c|c|c|c|c|c|c|c|c|}
\hline \multirow{4}{*}{ Actual } & Body & X center & Y center & Z center & dip & decl & strength & Cell Size \\
\cline { 2 - 9 } & 1 & 0 & 8 & -3 & 45 & 45 & 6 & 0.008 \\
\cline { 2 - 9 } & 2 & -8 & --8 & -2.5 & 80 & 120 & 7 & 0.008 \\
\hline \multirow{4}{*}{$\begin{array}{c}\text { True total } \\
\text { field }\end{array}$} & 1 & 0.02 & -8.02 & -2.95 & 45.0 & 44.6 & 24.6 & 0.002 \\
\cline { 2 - 9 } & 2 & -8.0 & -8.17 & -2.47 & 73.1 & 148 & 17.4 & 0.003 \\
\cline { 2 - 9 } & 3 & 7.97 & -8.02 & -1.99 & 36.5 & 71.1 & 17.1 & 0.003 \\
\hline \multirow{2}{*}{$\begin{array}{c}\text { Noisy total } \\
\text { field }\end{array}$} & 1 & -0.02 & 7.66 & -3.07 & 48.2 & 63.8 & 28.1 & 0.002 \\
\cline { 2 - 9 } & 2 & -8.0 & -8.02 & -2.47 & 77.9 & 132.0 & 17.4 & 0.003 \\
\cline { 2 - 9 } & 3 & 7.97 & -8.02 & -1.84 & 37.6 & 70.6 & 14.8 & 0.003 \\
\hline
\end{tabular}

\section{Conclusions}

We have incorporated the Euler Deconvolution Depth estimator and Magnetization vector Inversion to determine the locations as well as the internal magnetization vectors of buried magnetic objects. We have concluded that if the derivatives of total field are obtained accurately, the locations of objects can be well determined by combining Euler Deconvolution Depth estimator and some postprocess procedures. Upon obtaining Euler solutions, we may utilize them as the initial coarse locations and perform Magnetization Vector Inversion. It is also concluded that Magnetization Vector Inversion is relatively insensitive to data density and can work more stably. However, it has to be noticed that reasonably good Euler solutions can speed up Magnetization Vector Inversion dramatically. Starting with good Euler solutions is essential to guarantee an appropriate selection of dataset that incorporates substantial variation of the field and field gradients of each object.

We have utilized and modified a technique used in oil exploration for depth estimation and have shown that this can be useful. Our studies with real data are obviously more complicated as well as more interesting and some of these will be presented.

\section{References}

[1] R. W. Groom, R. Jia and C. Alvarez, Investigations into inversion of magnetic and gradient magnetic data for detection and discrimination of metallic objects, SAGEEP Meeting (2003).

[2] V. Mikehailov et al., Application of artificial intelligence for Euler solutions clustering, Geophysics, Vol. 68, NO.1, P.168-180, 2003.

[3] Reid,A.B., Allsop,J.M., Grancer,H., Millett,A.J., and Seomerton,I.W., Magnetic interpretation in three dimensions using Euler deconvolution: Geophysics, Vol. 55, P.80-91, 1990.

[4] Changyou Zhang, Martin F. Mushayandebvu, Alan B. Reid, J. Derek Fairhead, and Mark E. Odegard, Euler deconvolution of gravity tensor gradient data, Geophysics, Vol. 65, NO.2, P.512520,2000 